\title{
Family evaluations in acute intermittent porphyria using red cell uroporphyrinogen I synthetase ${ }^{1}$
}

\author{
JOEL M. LAMON, BRUCE C. FRYKHOLM, AND DONALD P. TSCHUDY \\ From the Metabolism Branch, National Cancer Institute, National Institutes of Health, \\ Bethesda, Maryland 20014, USA
}

SUMMARY Acute intermittent porphyria (AIP) is a primary disorder of haem biosynthesis that chemically characterised by raised urinary porphobilinogen (PBG). A defect in the biochemic pathway at the step of PBG conversion to uroporphyrinogen has been shown to be a result of $\vec{\Phi}$ partial deficiency of the enzyme uroporphyrinogen I synthetase (uro I syn). The ascertainment rate of latent AIP (that is, chemically manifest but clinically asymptomatic) was examined in 185 indivit duals from 12 AIP kindreds using three parameters: red cell uro I syn, quantitative urinary PBC and pedigree analysis with respect to uro I syn.

Approximately $80 \%$ of individuals could be assigned as normal or latent AIP on the basis of the uro I syn assay alone. The remaining $20 \%$ could not be assigned because of an intermediate range of activity for the red cell assay in which the diagnosis cannot be certain. When the pedigree was use in the evaluation of the uro I syn data, the number of uncertain individuals, with respect to AIRG decreased to $10 \%$. The enzyme method detected latent AIP in $37.5 \%$ of blood relatives, whereas quantitative urinary PBG alone detected only $15 \cdot 2 \%$. The pattern of inheritance for the uro I sy deficiency is consistent with Mendelian dominant inheritance, and it is likely that it is the base inherited defect in AIP.

Waldenström (1937) described the familial nature of acute intermittent porphyria (AIP) in Sweden. Autosomal dominant inheritance was suggested by this early study, which also showed that some asymptomatic family members excreted excess porphobilinogen in the urine. These chemically abnormal, but clinically asymptomatic, individuals were classified as having latent AIP. Later, HaegerAronsen (1958) suggested that the quantitative determination of urinary PBG and aminolaevulinic acid (ALA) would detect virtually all cases of latent AIP. Recently, a partial deficiency of approximately $50 \%$ of uroporphyrinogen I synthetase (uro I syn) has been associated with AIP (Strand et al., 1970). This deficient uro I syn activity has been shown in liver tissue (Strand et al., 1970), fibroblasts (Bonkowsky et al., 1975), lymphocytes (Sassa et al., 1977), and peripheral red cells (Magnussen et al., 1974) from patients with AIP.

Evaluation of blood relatives of clinically affected AIP individuals using the red cell uro I syn assay revealed a higher rate of latent AIP than would have

\footnotetext{
${ }^{1}$ Presented in part at the annual meeting of the American Society of Human Genetics, October 1977.

Received for publication 19 July 1978
}

been found using urinary PBG measurement alone It was the purpose of this study to assess the efficacy of these two diagnostic tests for the identification latent AIP in an affected kindred.

\section{Methods}

EXPERIMENTAL SUBJECTS

The kindreds of 12 AIP patients were evaluated using quantitative 24-hour urinary PBG and red ced uro I syn assay. The index case in each kindred had clinically manifest AIP. Their biochemical evaluag tion showed raised urinary PBG $(>2 \mathrm{mg} / 24$ hi $(8.8 \mu \mathrm{mol} / 24 \mathrm{~h})$ and deficient red cell uro I sygi activity ( $<23 \mathrm{nmol}$ porphyrin/ml RBC per $\mathrm{h}$ ). Reed cell samples were obtained from 185 individuals and urine specimens from 173 of those.

RED CELL UROPORPHYRINOGEN I SYNTHETASE Approximately $10 \mathrm{ml}$ heparinised blood was obtained by venipuncture. The red cells were washed with cold isotonic saline and the red cell pellet was stored $-4^{\circ} \mathrm{C}$. The assay was performed according to the method of Magnussen et al. (1974). Though a approximate $50 \%$ reduction in the uro $I$ syif 34 
activity is present in AIP, a range of activities for the red cell determination has been observed (Magnussen et al., 1974; Sassa et al., 1975; Bonkowsky and Bottomley, 1977). In this laboratory, the mean value of uro I syn for normals was $39 \cdot 8 \pm 7 \cdot 7$ (SD, $\mathrm{n}=37) \mathrm{nmol}$ porphyrin/ml RBC per $\mathrm{h}$ and $18 \cdot 6 \pm$ $3 \cdot 5(\mathrm{SD}, \mathrm{n}=17)$ for AIP patients who had increased urinary PBG. The intermediate or uncertain range of uro I syn activity was established using empirical limits of the highest uro I syn activity in a patient with raised urinary PBG for the upper limit, and the lowest uro I syn activity observed in a normal for the lower limit.

\section{QUANTITATIVE URINARY PBG}

The urinary PBG was measured from an aliquot of a 24-hour urine collection by the method of Mauzerall and Granick, as modified by Marver et al. (1966).

\section{Results}

The frequency distribution of red cell uro I syn activities among the family members, not including the index cases, is presented in Fig. 1. A bimodal distribution of enzyme activities, which would be expected in a disease with a $50 \%$ deficiency in the heterozygote, was not obvious. The possibility that interfamily variation in uro I syn activity might obscure such a distribution in the composite of 12 kindreds was considered, and Fig. 2 depicts the distribution of uro I syn activities in the individual kindreds. The index cases are included in that Fig. The suggestion that a bimodal distribution of uro I syn activity might occur within individual kindreds (Sassa et al., 1975) was not observed.

The ascertainment rate for latent AIP within kindreds at risk was $15 \cdot 2 \%$ when only the criterion of a raised urinary PBG was used. Deficient uro I syn activity ( $<23 \mathrm{nmol} / \mathrm{ml} \mathrm{RBC}$ per h) was observed in $37.5 \%$ of blood relatives tested. The improvement in ascertainment of latent AIP using only the red cell uro I syn assay instead of urinary PBG was significant $(P<0 \cdot 0005)$ by the $\chi^{2}$ test. The ascertainment rate using the uro I syn assay was improved by only $1.6 \%$ when urinary PBG information was also used (Fig. 3). This additional $1.6 \%$ represents individuals with increased urinary PBG in addition to a uro I syn greater than $23 \mathrm{nmol}$ porphyrin $/ \mathrm{ml}$ RBC per $h$. Using an evaluation of the pedigree with respect to the uro I syn activities improved the assignment of family members as normal with respect to AIP, and lowered the frequency of uncertain individuals by a half (Fig. 3).

Using the concepts of simple Mendelian dominant inheritance, the pedigrees were evaluated in two ways: (1) by the use of parents' uro I syn activity to classify an offspring with intermediate uro I syn activity into either normal or porphyria groups; and (2) by the use of a clearly porphyric uro I syn activity in an offspring to clarify the classification of a parent with intermediate uro I syn activity. Intermediate uro I syn activity $(>23$ and $<30 \mathrm{nmol}$ porphyrin/ml $\mathrm{RBC}$ per $\mathrm{h}$ ) in offspring whose

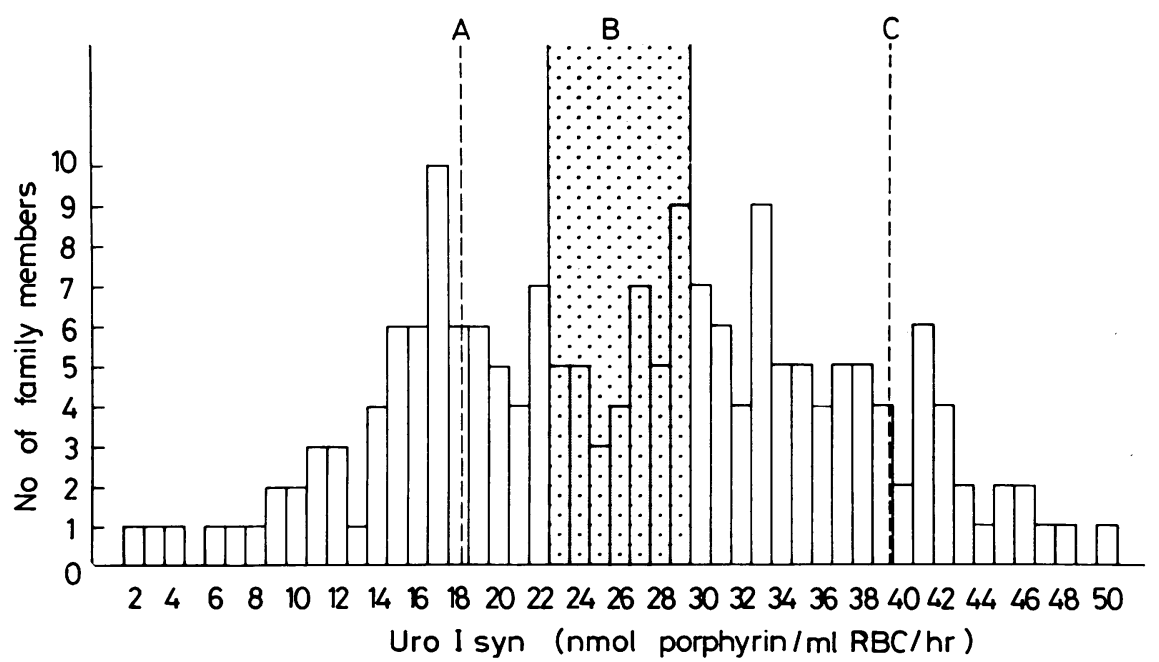

Fig. 1 Frequency distribution of red cell uro I syn activity in AIP family members. A, mean uro I syn for manifest AIP cases, $18.6 \pm 3.5 \mathrm{nmol}$ porphyrin $/ \mathrm{ml} R B C$ per $h(n=17)$. $B$, intermediate range of uro I syn activity, $>23$ and $<30 \mathrm{nmol}$ porphyrin/ml $R B C$ per $h$. C, mean uro I syn for normals, $39.8 \pm 7.7 \mathrm{nmol}$ porphyrin/ml RBC $\operatorname{per} h(n=37)$. 


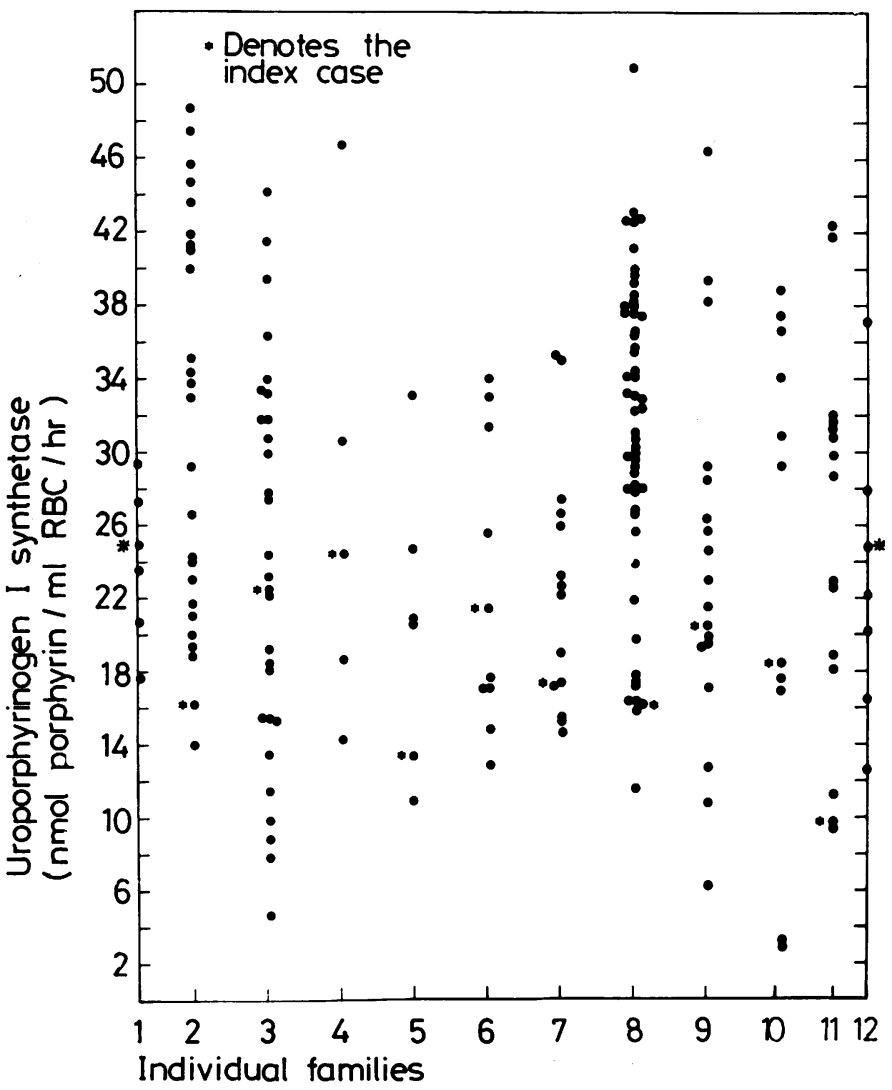

Fig. 2 Distribution of uro I syn activit禺 in the 12 AIP kindreds studied.

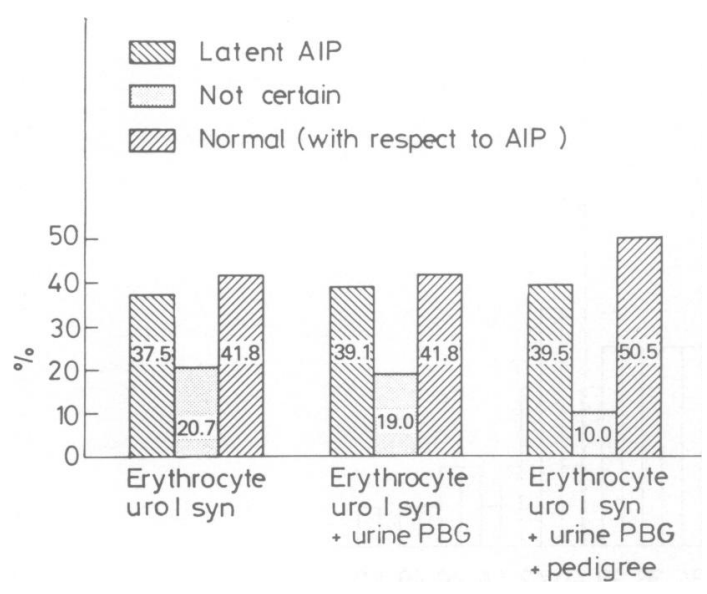

Fig. 3 Percentage of total cases (185) assigned as latent AIP, not certain, or normal, via three means of evaluation: (1) uro I syn activity only; (2) uro I syn activity plus 24-hour urinary $P B G$; and (3) uro I syn pedigree, as illustrated in Fig. 6. parents had unequivocally normal activity werè considered normal; intermediate uro I syn activit危 in a parent of an individual with deficient activit女 was classified as latent AIP. The combination of. normal uro I syn in both parents and deficient uro $\mathrm{g}$ syn activity in a child of these parents was no未 observed. An example of this approach can be seem in the pedigree (Fig. 4). Fig. 3 shows that this pedigree evaluation of uro I syn activity was mos\$ helpful in assigning uncertain individuals as norma with respect to AIP.

Of the 12 index cases in this study, 11 were femalen It is well known that manifest AIP is more frequentu in females. Though the percentage in the intermediate group differed considerably from males to females the ratio of porphyric to normal was compatible with Mendelian dominant inheritance of this defecten The detection rate of latent AIP by uro I syn measurement appears to be higher in females thar in males (Fig. 5). Normal, deficient, and intermediat uro I syn activities were observed in family member of all ages, but no family member below the age of 


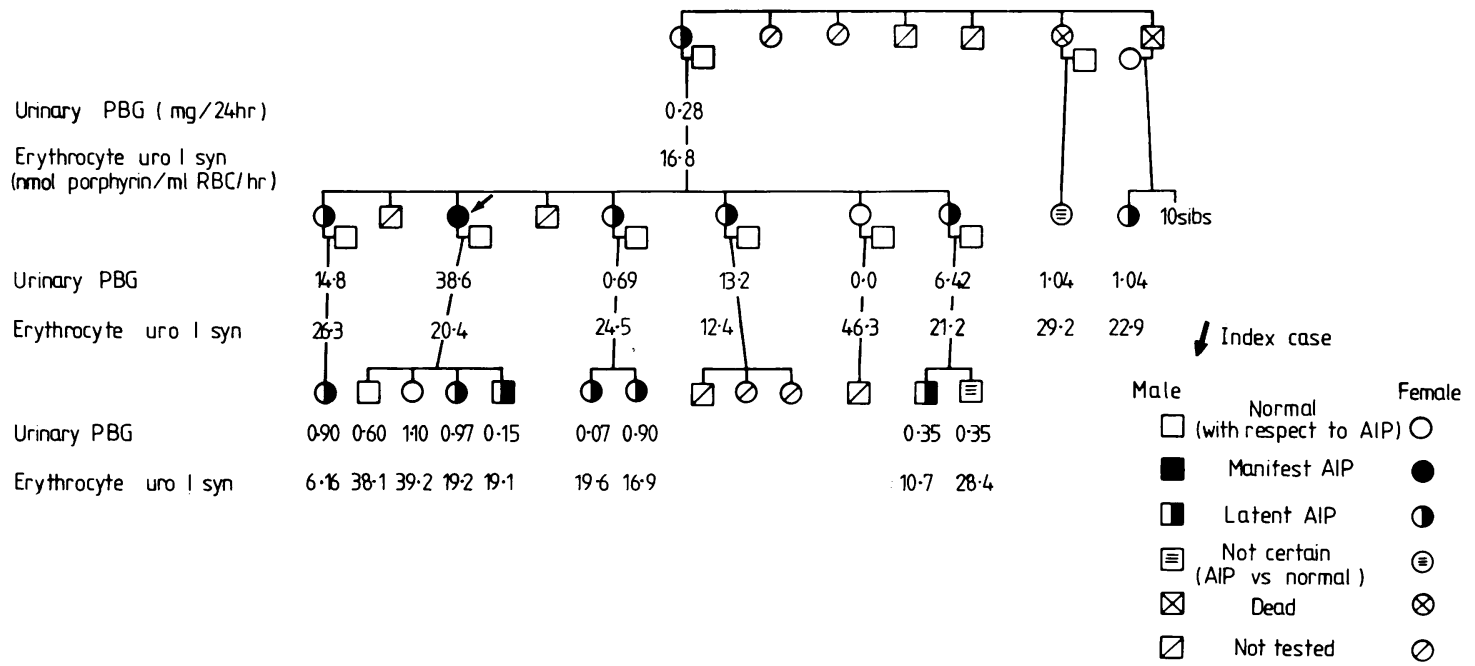

Fig. 4 Pedigree of one of the 12 families studied. Red cell uro I syn and urinary PBG data are listed below the individual tested.

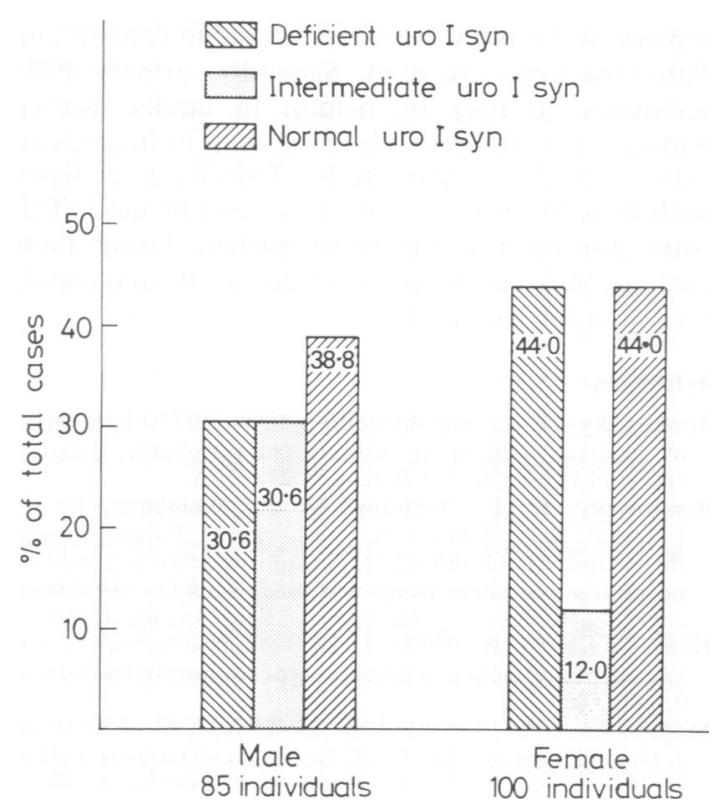

$100 \%$ of those above the age of 24 had increased PBG. Of 24 samples of urine positive for PBG, 6 were found in males.

Fig. 5 Sex distribution of latent AIP and normal individuals. Percentage of total cases assigned as latent AIP or normal on the basis of uro I syn assay only.

24 excreted excess urinary PBG (Fig. 6). In Fig. 6 it can be seen that there is a striking contrast between the correlation of lowered enzyme and increased urinary PBG in individuals under the age of 24 , as compared with those above this age. At enzyme levels below $15 \mathrm{nmol}$ porphyrin/ml RBC per h,

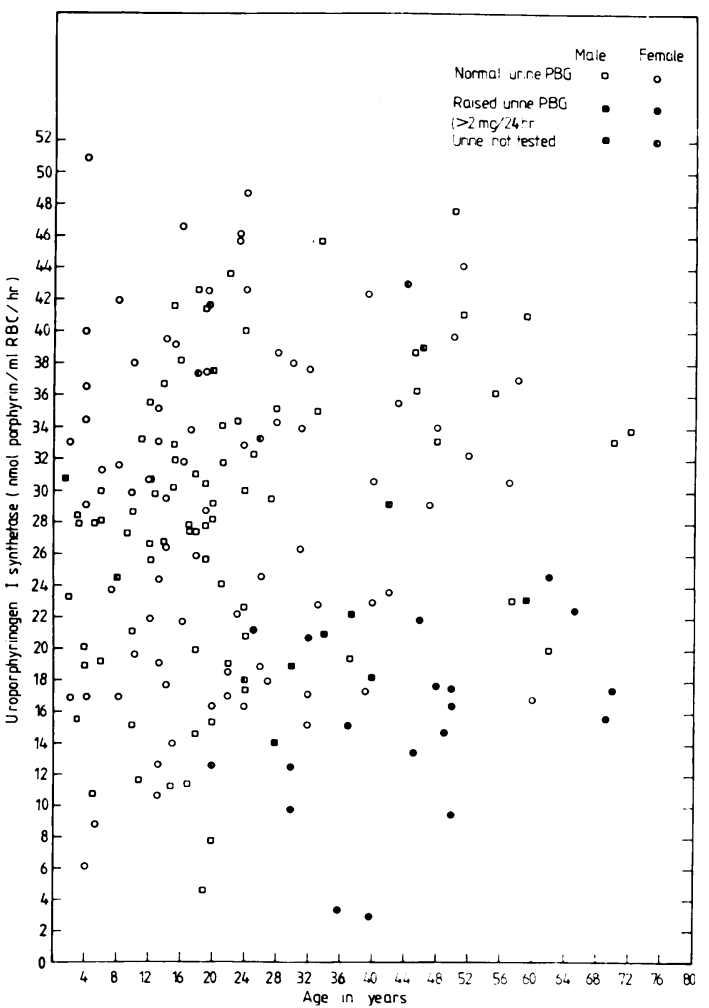

Fig. 6 Age vs. uro I syn activity. 


\section{Discussion}

Demonstration of raised urine PBG excretion during the acute attack interval of AIP, as well as variegate and hereditary coproporphyria, continues to be the fundamental diagnostic parameter in the acute attack types of porphyria. Previously, quantitative 24-hour urinary PBG determination was the only objective parameter available to attempt to ascertain latent AIP within a kindred. This study confirms the impression that the measurement of uro I syn activity in an AIP kindred identifies a significantly larger number of individuals with latent AIP than the urine PBG evaluation.

The hypothesis that a partial deficiency of uro I syn might be the basic inherited defect in AIP is supported by the current study. Waldenström (1937) postulated autosomal dominant inheritance for AIP based upon pedigree analysis of clinically affected and latent AIP (defined by raised urinary PBG) patients. Though more latent AIP cases were identified within these kindreds by uro I syn assay as compared to urine PBG measurement alone, the pattern of inheritance for the uro I syn deficiency remained consistent with Mendelian dominant inheritance. The equal distribution of normal and latent AIP among all family members (Fig. 3), and the approximately equal sex ratio (Fig. 5) using the uro I syn data, also support that hypothesis.

The identification of a partial deficiency of uro I syn as the probable inherited defect in AIP has not improved the understanding of the pathogenesis of the clinically manifest disease. Specifically, the reasons why only some individuals with deficient uro I syn activity have increased urinary PBG (15 of 36 individuals in this study), and not all of those become symptomatic, are not known. Since all family members under the age of 24 were negative for urine PBG, as seen in Fig. 6, great decreases in the level of red cell uro I syn alone do not produce either chemical (increased urinary PBG) or clinical manifestations of the disease. This was the case even in the face of very low levels of the enzyme in some of these individuals. It has been known that children virtually never manifest the disease chemically or clinically. The present study not only confirms this, but shows that the difference between children and adults cannot be attributed to differences in enzyme level, at least with regard to the erythrocyte. Whether there are age differences in the activity of the liver enzyme is unknown. The present study further supports previous evidence that other factors, presumably hormonal, are necessary for the chemical and clinical manifestations of the disease (Tschudy, 1974). It would appear, therefore, that two conditions, the inherited deficiency of uro I syn and adulthood, are necessary but not sufficient con $\frac{\mathbb{}}{\mathcal{D}}$ ditions for clinical activity of the disease. Clinica experience has shown that an acute exacerbation requires other factors in addition to these conditions? (Tschudy, 1974). These factors include certain medications, starvation, infections, and an associa음. tion with menstrual cycles in some females (Tschudy? et al., 1975). Therefore, the identification of latento AIP individuals who may be at risk of developings an acute attack is important for the prevention of unnecessary morbidity and mortality.

It is clear from this investigation that $80 \%$ of individuals in an AIP kindred can be classified as? either latent AIP or normal by using red cell uro $\overline{\mathrm{B}}$ syn assay. Quantitative 24-hour urinary PBG. determinations on family members do not signi- $\Omega$ ficantly improve the ascertainment of latent AIP beyond that achieved by the enzyme determination $\omega$ alone. For the approximately $20 \%$ of familye members with uncertain uro I syn values, three approaches to achieve conclusive data are possible $>$ Firstly, one can repeat the assay taking care that the reticulocyte count is not raised, that the blood sampleis processed rapidly, and that adequate control and blank reactions are used. Secondly, urinary PBG $^{6}$ determination may be helpful in adults. Raised urinary PBG has been observed only in individuals older than 24 in this study. Thirdly, a pedigrees analysis with respect to uro I syn can be used. Thiš latter approach is described earlier. Using these methods $90 \%$ of an AIP kindred can be adequately $\overrightarrow{5}$ evaluated for latent AIP.

\section{References}

Bonkowsky, H. L., and Bottomley, S. S. (1977). Evaluation of kindreds with acute intermittent porphyria: progres and problems. Clinical Research, 25, 517A.

Bonkowsky, H. L., Tschudy, D. P., Weinbach, E. C. Ebert, P. S., and Doherty, J. M. (1975). Porphyrin sync thesis and mitochondrial respiration in acute intermitten? porphyria. Studies using cultured human fibroblasts Journal of Laboratory and Clinical Medicine, 85, 93-102. 응

Haeger-Aronsen, B. (1958). Urinary $\delta$-aminolaevulinic acid and porphobilinogen in different types of porphyria. Lance $\frac{7}{8}$ 2, 606-608.

Magnussen, C. R., Levine, J. B., Doherty, J. M., Chessman J. O., and Tschudy, D. P. (1974). A red cell enzyme methodr for the diagnosis of acute intermittent porphyria. Blood 44, 857-868.

Marver, H. S., Tschudy, D. P., Perlroth, M. G., Collins, A.స్ and Hunter, G., Jr. (1966). The determination of aminoE ketones in biological fluids. Analytical Biochemistry, 14 53-60.

Sassa, S., Granick, S., and Kappas, A. (1975). Effect of lead and genetic factors on heme biosynthesis in the human rect cell. Annals of the New York Academy of Sciences, 244,0 419-440.

Sassa, S., Zalar, G. L., and Kappas, A. (1977). Deficien⿳亠口冋 induction of uroporphyrinogen-I synthetase (URO-S activity in mitogen-stimulated lymphocytes from acut\& intermittent porphyria (AIP). Clinical Research, 25, 517A] 
Strand, L. J., Felsher, B. F., Redeker, A. G., and Marver, H. S. (1970). Heme biosynthesis in intermittent acute porphyria. Decreased hepatic conversion of porphobilinogen to porphyrins and increased delta-aminolevulinic acid synthetase activity. Proceedings of the National Academy of Sciences of the U.S.A., 67, 1315-1320.

Tschudy, D. P. (1974). Porphyrin metabolism and the porphyrias. In Duncan's Diseases of Metabolism, pp. 775824. Ed. by P. K. Bondy, and L. E. Rosenberg. Saunders, Philadelphia.

Tschudy, D. P., Valsamis, M., and Magnussen, C. R. (1975).
Acute intermittent porphyria: clinical and selected research aspects. Annals of Internal Medicine, 83, 851-864.

Waldenström, J. (1937). Studien uber porphyrie. Acta Medica Scandinavica, Suppl. 82, 1-254.

Requests for reprints to Dr Joel M. Lamon, Clinical Epidemiology Branch, National Cancer Institute, A521 Landow Building, National Institutes of Health, Bethesda, Maryland 20014, USA. 